\title{
Migraine headaches in a nutshell
}

\author{
N Schellack, ${ }^{1}$ O Mogole, ${ }^{2}$ N Magongwa, ${ }^{2}$ F Makola ${ }^{2}$ \\ ${ }^{1}$ Associate Professor, School of Pharmacy, Faculty of Health Care Sciences, Sefako Makgatho Health Sciences University \\ ${ }^{2}$ Academic Intern, School of Pharmacy, Faculty of Health Care Sciences, Sefako Makgatho Health Sciences University \\ *Corresponding author, email: natalie.schellack@smu.ac.za
}

\begin{abstract}
This article aims to provide a concise, high-level overview of the classification, management and treatment of migraine. Migraine is a common, debilitating neurological disorder that is characterised by the presence of severe headaches, which may last anything from a few hours to a few days (4-72 hours). Thus, the condition is characterised by episodes of severe migraine headache, frequently accompanied by nausea and vomiting. These headaches may be unilateral or bilateral, and patients may also experience a range of associated features. Acute attacks require rapid, abortive treatment and the rate of recurrence needs to be reduced and managed using effective prophylactic measures.
\end{abstract}

Keywords: migraine headache, aura, ergot alkaloids, triptans

\section{Introduction}

According to the International Headache Society, a migraine is a headache that lasts for 4-72 hours and presents with at least two of the following symptoms: unilateral localisation, moderate to severe pain intensity, aggravation by movement, and a pulsating feeling. The headache is also usually accompanied by nausea, vomiting, photo- and phonophobia. ${ }^{1}$ Migraine headaches are typically classified into two major subtypes, namely migraine with an aura, and a migraine headache without an aura. ${ }^{2}$ Migraine is considered to be chronic when it occurs for a minimum duration of four (4) hours per day, and lasts for more than 15 days per month, within a three-month period. ${ }^{3}$ Chronic migraine is frequently associated with the so-called medicationoveruse-headache. $^{4}$

A migraine headache is generally preceded by a premonitory phase that lasts for hours before the headache begins. ${ }^{1}$ This phase is characterised by fatigue, mood changes and gastrointestinal problems, which could persist throughout the entire migraine attack. ' One in five 'migraineurs' (i.e. people who suffer from migraine) also experience an aura, which consists of visual, sensory or motor disturbances. ${ }^{1}$ The aura phase is followed by actual headache and this, in turn, is followed by a recovery phase, or postdrome (also referred to as a 'migraine-hangover'), with fatigue and continued sensory disturbances. ${ }^{5}$

\section{Pathophysiology}

Migraine headaches have a controversial pathophysiology. The most widely acceptable pathophysiological process involves the activation and sensitisation of the trigeminovascular system
(TVS). ${ }^{6}$ When the TVS is activated, the signal travels through the trigeminal ganglion to the neurons in the trigeminocervical complex, with calcitonin gene-related peptide (CGRP) as the main neurotransmitter. ${ }^{7}$ CGRP is a potent vasodilator, produced

\begin{tabular}{|l|l|}
\hline Migraine & $\begin{array}{l}\text { - At least five attacks } \\
\text { without aura }\end{array}$ \\
- The attack lasts for four to 72 hours (untreated or \\
- Unsuccessfully treated) \\
- Pulsating quality \\
- Moderate to sever intensity \\
- Headache is aggravated by physical activity \\
- The following may co-exist with the headache: \\
nausea and/or vomiting, photophobia and \\
phonophobia
\end{tabular}

\begin{tabular}{|l|l|}
\hline Migraine & $\begin{array}{l}\text { - 'Classic migraine' } \\
\text { - At least two attacks }\end{array}$ \\
- The aura fulfills the criteria for typical aura, \\
hemiplegic aura or basilar-type aura \\
- Aura refers to a neurological symptom that \\
precedes the attack and in some instances \\
accompanies the attack \\
- Not attributable to another condition
\end{tabular}

\begin{tabular}{|l|l|}
\hline - Reversible, might be visual, sensory or speech \\
symptoms with no motor weakness
\end{tabular}

Figure 1: Subtype classifications of migrain $e^{10}$ 
in the central and peripheral neurons, that has been implicated in the transmission of pain signals and is released during severe migraine attacks. ${ }^{8}$

\section{Classification}

Migraine is usually classified as having two major subtypes, namely migraine with an aura, and migraine without an aura. Additional subtype classifications are depicted in Figure 1.

\section{Diagnosis}

The diagnosis is made, based on the clinical presentation or symptoms of the patient, and by excluding other causes of frequent headaches. ${ }^{9}$ In many cases the pain experienced during migraine attacks, occurs unilaterally. ${ }^{10}$ The pain is described as severe and is accompanied by nausea and/or vomiting, hypersensitivity to light, sound and odour. ${ }^{7}$

Chronic migraine diagnosis relies on the International Classification of Headache Disorders (ICHD-3) beta criteria, because there are no biological markers for chronic migraine. ${ }^{3}$

Table I: International Classification of Headache Disorders diagnostic criteria for migraine ${ }^{10}$

1. At least 5 attacks fulfilling criteria $2-4$
2. Headache attacks lasting $4-72$ hours
3. Headache has at least two of the following four characteristics
a. Unilateral location
b. Pulsating quality
c. Moderate or severe pain intensity
d. Aggravation by or causing avoidance of routine physical
activity

4. During headache, at least one of the following:

a. Nausea and/or vomiting

b. Photophobia and phonophobia

5. Not better accounted for by another ICHD-3 diagnosis

\section{Pharmacological management}

From a pharmacotherapeutic perspective, migraine management may be divided into prophylactic measures, and the treatment of acute attacks, as described in the following section.

\section{Migraine prevention}

Migraine attacks can range in intensity from moderate to severe, and may be preceded by other symptoms. ${ }^{8}$ The migraines can change from being episodic to being chronic. Although episodic migraine can remain unchanged for years, there is also the likelihood that it could remit or develop into a situation whereby it may be classified as chronic, with an increasing severity and frequency of headaches per month. ${ }^{8}$ When the headaches do become chronic, it would be highly advisable to look the migraineur in question and individualise preventative migraine therapy. ${ }^{8}$ These drugs will, on average, reduce migraine frequency by $50 \%$ in about $40-45 \%$ of patients; however, compliance and adherence are poor because of their many adverse effects. Drug classes for migraine prevention are described below.

\section{$\beta$-blockers}

The following beta-blockers have proven efficacy in this setting: atenolol, metoprolol, nadolol, propranolol and timolol. ${ }^{2}$ Use of these drugs should be carefully monitored in patients who exhibit undesirable adverse effects and switched to a different class, such as the anti-epileptic agents (e.g. valproic acid). ${ }^{2}$

\section{Anti-epileptic agents}

Several anti-epileptics have shown increasing potential in migraine prevention. Treatment options include carbamazepine, valproate, gabapentin, topiramate and lamotrigine. ${ }^{2}$ In this class, topiramate is one of the most effective therapy options to consider in patients with chronic migraine. ${ }^{2}$

\section{Serotonin-receptor antagonists}

Methysergide is a $5-\mathrm{HT}_{2}$ receptor antagonist and $5-\mathrm{HT}_{1 \mathrm{~B} / \mathrm{D}}$ receptor agonist, and pizotifen, a $5-\mathrm{HT}_{2}$ receptor antagonist ${ }^{15}$. Both drugs provide adequate and effective prophylaxis against migraines. ${ }^{15}$ Currently, pizotifen is registered for use in South Africa but mainly considered for use in patients that are refractory to other prophylactic drugs, due to the high cardiac adverse effect profile. The use of pizotifen should not be longer than six months. ${ }^{15}$

\section{Other agents}

Flunarizine is a calcium channel blocker that also has antihistaminergic properties. It is used for migraine prophylaxis and in some instances, as a first line drug in children with hemiplegic migraines. ${ }^{16}$ Other calcium-channel blockers include verapamil.2 Angiotensin II-receptor antagonists (e.g. candesartan), and certain antidepressants (e.g. amitriptyline and venlafaxine), are other potential options. ${ }^{2}$

\section{Botulinum toxin $A$}

Onabotulinum toxin $\mathrm{A}$ is classified as a neurotoxin, which is primarily a product of the anaerobic bacterium, Clostridium botulinum. The toxin appears to exert its mechanism of action by inhibiting the release of nociceptive mediators involved in the pathogenesis of migraine. ${ }^{4}$ These include substance $P$, CGRP and glutamate; it inhibits these nociceptive mediators from the peripheral nerve terminals of primary afferents. ${ }^{4}$ In the two randomised clinical trials that the drug underwent, it was evident from the data collected that onabotulinum toxin $A$ is a safe, well-tolerated, and an effective prophylactic treatment in patients suffering from chronic migraine. ${ }^{3}$

Table II: Classes and examples of drugs that are used in the prevention of migraine headaches in adults, in South Africa ${ }^{2}$

\begin{tabular}{ll}
\hline CLASS & EXAMPLES \\
\hline Beta-blockers & Atenolol \\
& Metoprolol \\
& Nadolol \\
& Propranolol \\
& Timolol \\
& Carbamazepine \\
Anti-epileptic agents & Valproate \\
& Gabapentin \\
& Topiramate \\
& Lamotrigine \\
\hline Calcium-channel blockers & Verapamil \\
\hline Angiotensin II-receptor antagonists & Candesartan \\
\hline Antidepressants & Amitriptyline \\
\hline
\end{tabular}




\section{Managing acute attacks}

Acute migraine attacks should be treated early, when the pain is still mild and current guidelines recommend non-steroidal antiinflammatory drugs (NSAIDs), aspirin and paracetamol for the treatment of acute mild to moderate migraine attacks. ${ }^{9}$ Many of the drugs used for the treatment of acute migraine attacks are also used for treating chronic migraine. ${ }^{9}$

Ergot alkaloids, such as ergotamine, are 5-HT-receptor agonists and bind to a-adrenoceptors and dopamine receptors. ${ }^{4}$ Their use has been reduced since the arrival and introduction of the triptans. ${ }^{4}$ Their use has also been declining due to their unwanted side-effects, inconvenience and a high likelihood for causing medication-overuse headaches. ${ }^{2}$

Sumatriptan was specifically formulated for the treatment of acute migraine attacks. It has a high specificity for the $5-\mathrm{HT}_{1 \mathrm{~B}}$ and $5-\mathrm{HT}_{1 \mathrm{D}}$ serotonin receptors. ${ }^{2}$ This mechanism of action produces cerebral vasoconstriction, secondary to their inhibition of calcitonin gene-related peptide (CGRP) and inflammatory peptide release. ${ }^{2}$ The triptans are most effective when they are taken when the pain is mild to moderate. ${ }^{8}$ There are a few more triptans that have been introduced for use in South Africa, including zolmitriptan, naratriptan, rizatriptan and elitriptan. ${ }^{2}$

Table III: NSAIDS used for acute migraine treatment in adults ${ }^{10}$

\begin{tabular}{ll}
\hline NSAIDS & DOSAGE \\
\hline Paracetamol & 1 gram \\
Aspirin & $900-1200 \mathrm{mg}$ \\
Ibuprofen & $400-800 \mathrm{mg}$ \\
Naproxen & $250-500 \mathrm{mg}$ \\
\hline
\end{tabular}

Table IV: Triptans used for acute migraine treatment in adults ${ }^{10}$

\begin{tabular}{ll}
\hline TRIPTAN DRUGS & DOSAGE \\
\hline Sumatriptan & $\begin{array}{l}50-100 \mathrm{mg} \text { orally, 10-20 mg nasal, } 6 \\
\mathrm{mg} \text { subcutaneously }\end{array}$ \\
\hline Almotriptan & $12.5 \mathrm{mg}$ \\
\hline Eletriptan & $40-80 \mathrm{mg}$ \\
\hline Frovatriptan & $2.5 \mathrm{mg}$ \\
\hline Naratriptan & $2.5-5 \mathrm{mg}$ \\
\hline Rizatriptan & $5-10 \mathrm{mg}, \mathrm{s} / \mathrm{l}$ melt \\
\hline Zolmitriptan & $5-10 \mathrm{mg}$ orally, s/l melt, $5 \mathrm{mg}$ nasal \\
\hline
\end{tabular}

The concomitant use of other agents that increase serotonin levels, such as the SSRIs and the serotonin-noradrenaline reuptake inhibitors (SNRIs), should be avoided due to the danger of developing serotonin syndrome. ${ }^{11}$ The triptans should also be avoided in patients with a history of ischemic heart disease, cerebrovascular disease and uncontrolled hypertension. ${ }^{12}$

A novel, highly-selective 5- $\mathrm{HT}_{1 \mathrm{~F}}$-receptor agonist, with a seemingly good cardiovascular safety profile, namely lasmiditan, is currently in clinical development. Two CGRP antagonists have also shown promise during clinical development, namely olcegepant and telcagepant. ${ }^{13}$ The latter has, however, been discontinued due to safety concerns. ${ }^{14}$

\section{Conclusion}

Migraine headaches are commonly encountered in the clinical practice setting. Patients suffering from migraine must endure an often-debilitating neurological disorder, with frequent attacks of severe headache that require acute, abortive treatment. The recurrence of these episodes may also be markedly decreased using effective, preventive measures, including the use of prophylactic medication. The clinician may play a vital role in the effective management of migraine through the promotion of a better understanding of the correct and effective use of migraine treatment options, as well as the reduction and management of associated risk factors and behaviours.

\section{References}

1. Russo AF. Calcitonin Gene-Related Peptide (CGRP): A New Target for Migraine. Annu Rev Pharmacol Toxicol. 2015;55(1):533-52. doi: 10.1146/ annurev-pharmtox-010814-124701

2. Schellack N, Schellack G. An Overview of Migraine Management and Treatment. S Afr Pharm J. 2013;80(9):26-31.

3. Carod-Artal FJ. Tackling Chronic Migraine: Current Perspectives. J Pain Res. 2014; 185. doi: 10.2147/JPR.S61819

4. Roceanu A, Antochi F, Bajenaru O. New molecules in migraine treatment. Farmacia. 2015;63(4):475-81.

5. Migraine Phases. 2017 [accessed on 5 Apr 2017]. Available from: https:// migraine.com/migraine-basics/migraine-phases

6. Espinosa-Sanchez JM, Lopez-Escamez JA. New Insights into Pathophysiology of Vestibular Migraine. Frontiers in Neurology. 2015;6:n pag. Available from: https:// doi.org/10.3389/fneur.2015.00012

7. Ferrari MD, et al. Migraine Pathophysiology: Lessons from Mouse Models and Human Genetics. The Lancet Neurology. 2015;14.(1): 65-80. Available from: http://dx.doi.org/10.1016/S1474-4422(14)70220-0

8. Diener HC, et al. New Therapeutic Approaches for The Prevention and Treatment of Migraine. The Lancet Neurology. 2015;14(10): 1010-22. Available from: http:// dx.doi.org/10.1016/S1474-4422(15)00198-2

9. Schwedt TJ. Chronic Migraine. BMJ. 24 Mar2014:348(5):g1416-g1416. doi: 10.1136/bmj.g1416

10. Weatherall MW. The Diagnosis and Treatment of Chronic Migraine. Therapeutic Advances in Chronic Disease. 2015;6(3):115-23. doi: https://doi. org/10.1177/2040622315579627

11. Tepper SJ, Spears RC. Acute treatment of migraine. Neurol Clin. 2009;27:417-27. Available from: http://dx.doi.org/10.1016/j.ncl.2008.11.008

12. Minor DS, Wofford MR. Headache disorders. In: Pharmacotherapy: a pathophysiological approach. DiPiro JT, et al., eds. 7th ed. New York: McGraw-Hill Medical; 2008.

13. Magis D, Schoenen J. Treatment of migraine: update on new therapies. Curr Opin Neurol. 2011;24:203-10. doi: 10.1097/WCO.0b013e3283462c3f

14. Durham PL, Vause CV. CGRP receptor antagonists in the treatment of migraine. CNS Drugs. 2010;24(7): 539-48. doi:10.2165/11534920-000000000-00000

15. Schürks $M$, Diener $H$, Goadsby $P$. Update on the prophylaxis of migraine. Curr Treat Options Neurol. 2008;10(1):20-29.

16. Mohamed BP, Goadsby PJ, Prabhakar P. Safety and efficacy of flunarizine in childhood migraine: 11 years' experience, with emphasis on its effect in hemiplegic migraine. Dev Med Child Neurol. 2012;54(3):274-7. 